\title{
Two novel species of Vagicola (Phaeosphaeriaceae) from Italy
}

\section{Jayasiri $\mathrm{SC}^{1}$, Wanasinghe $\mathrm{DN}^{1,2}$, Ariyawansa $\mathrm{HA}^{3}$, Jones $\mathrm{EBG}^{4}$, Kang $\mathrm{JC}^{5}$, Promputtha $\mathrm{I}^{6}$, Bahkali $\mathrm{AH}^{4}$, Bhat $\mathrm{J}^{7,8}$, Camporesi $\mathrm{E}^{9}$ and ${\mathrm{Hyde} \mathrm{KD}^{1,2,4}}^{1,4}$}

\author{
${ }^{1}$ Center of Excellence in Fungal Research, Mae Fah Luang University, Chiang Rai 57100, Thailand \\ ${ }^{2}$ World Agro forestry Centre East and Central Asia Office, 132 Lanhei Road, Kunming 650201, China \\ ${ }^{3}$ Guizhou Key Laboratory of Agricultural Biotechnology, Guizhou Academy of Agricultural Sciences, Guiyang, 550006, \\ Guizhou, China \\ ${ }^{4}$ Botany and Microbiology Department, College of Science, King Saud University, Riyadh, 1145, Saudi Arabia \\ ${ }^{5}$ Engineering Research Center of Southwest Bio-Pharmaceutical Resources, Ministry of Education, Guizhou \\ University, Guiyang 550025, Guizhou Province, China \\ ${ }^{6}$ Department of Biology, Faculty of Science, Chiang Mai University, Chiang Mai, 50200, Thailand \\ ${ }^{7}$ No. 128/1-J, Azad Housing Society, Curca, P.O. Goa Velha, 403108, India \\ ${ }^{8}$ Department of Botany, Goa University, Goa, 403 206, India \\ ${ }^{9}$ A.M.B. GruppoMicologicoForlivese "Antonio Cicognani", Via Roma 18, Forli, Italy; A.M.B. CircoloMicologico \\ “Giovanni Carini”, C.P. 314, Brescia, Italy; Società per gliStudiNaturalisticidella Romagna, C.P. 144, Bagnacavallo \\ (RA), Italy
}

Jayasiri SC, Wanasinghe DN, Ariyawansa HA, Jones EBG, Kang JC, Promputtha I, Bahkali AH, Bhat J, Camporesi E, Hyde KD 2015 - Two novel species of Vagicola (Phaeosphaeriaceae) from Italy. Mycosphere 6(6), 716-728, Doi 10.5943/mycosphere/6/6/7

\begin{abstract}
Phaeosphaeriaceae is a large and important family in the order Pleosporales, comprising economically important plant pathogens. Species may also be endophytes or saprobes on plant hosts. Two new species referable to Vagicola, Phaeosphaeriaceae are introduced in this paper based on analyses of LSU and ITS sequence data and their unique morphology. Most Phaeosphaeriaceae species grow on monocotyledons; Vagicola dactylidis and V. chlamydospora are also saprobic on grasses (Poaceae). Vagicola chlamydospora formed asexual structures in a culture. The new species are described and illustrated and compared with other taxa.
\end{abstract}

Key words - LSU - ITS - monocotyledons - multigene analyses - Poaceae

\section{Introduction}

Phaeosphaeriaceae is a large family in the order Pleosporales (Hyde et al. 2013, Phookamsak et al. 2014). Members of this group grow mainly on monocotyledons, but some species have also been reported on dicotyledons (Shoemaker and Babcock 1989, Schoch et al. 2006, Zhang et al. 2009, 2012, De Gruyter et al. 2010, Hyde et al. 2013, Wijayawardene et al. 2014). The family was introduced by Barr (1979) and recent studies have shown it to be a natural group comprising 25 genera (Ariyawansa et al. 2015). Ariyawansa et al. (2015) and Phukhamsakda et al. (2015) have provided the latest backbone trees for the family. The asexual morphs are coelomycetous (Zhang et al. 2009, Phookamsak et al. 2014, Wijayawardene et al. 2014, Li et al. 2015). The family Phaeosphaeriaceae has a cosmopolitan distribution, and species are generally 
necrotrophic, plant pathogens or saprobes on a wide range of plants Shoemaker and Babcock 1989, Carson 2005, Stukenbrock et al. 2006, Cannon and Kirk 2007.

The genus Phaeosphaeria was introduced by Miyake (1909). Miyake (1909) treated 114 species of Phaeosphaeria and accommodated them in six subgenera, viz. Ovispora, Fusispora, Phaeosphaeria, Spathispora, Vagispora and Sicispora based on the differences in ascospore shape, number of septa and the gelatinous sheaths on spores (Eriksson 1967, Shoemaker \& Babcock 1989). The morphological characters of taxa in this genus are often ambiguous and can be confused with other taxa in the Leptosphaeriaceae and Montagnulaceae, and with genera in the family itself (Hyde et al. 2013, Phookamsak et al. 2014). Multigene phylogenetic analyses were carried out to confirm the placement of this group by Zhang et al. (2009), Phookamsak et al. (2014) and Ariyawansa et al. (2015). In this paper, we introduce two new species in Vagicola from Italy, which were found on dead culms of Dactylis sp. (Poaceae). Combined analyses of LSU and ITS sequence data using maximum-likelihood (ML) and maximum-parsimony (MP) clearly showed these species grouped in Phaeosphaeriaceae with strong statistical support. In this paper, the two new species are described and illustrated and compared with similar taxa.

\section{Material and Methods}

\section{Collections, morphology and isolation}

Specimens were collected in Italy by Erio Campesori. Study of gross morphology and photomicrography were carried out under a stereomicroscope. Sections of ascoma were made freehand. Several specimens were used to observe the asci and ascospore characters and slides were preserved in lactoglycerol. Micro-morphological characters were observed under a compound microscope (Nikon Eclipse Ni), and measurements made using Tarosoft (R) Image Frame Work v. 0.9.7. Single spore isolation was carried out following the method of Chomnunti et al. (2014). Type specimens of the new species are deposited in the Mae Fah Luang University Herbarium (MFLU), Chiang Rai, Thailand, and ex-type cultures in Mae Fah Luang University Culture Collection (MFLUCC) and Kunming Institute of Botany (KIB). Facesoffungi numbers and Index Fungorum numbers are as outlined in Jayasiri et al. (2015) and Index Fungorum (2015).

\section{Establishing the asexual morphs}

Circular $(0.5 \mathrm{~cm})$ agar blocks from growing colony margins were cut and placed on fresh Malt Extract Agar (MEA) plates as described in Phooksamak et al. (2015). Asexual structures produced on Malt Extract Agar were observed after eight weeks of incubation, under light, at $20^{\circ}$ C.

\section{DNA isolation, amplification and sequencing}

Fungal isolates were grown on $2 \%$ MEA for 20 days at $16^{\circ} \mathrm{C}$. Genomic DNA was extracted from the growing mycelium using the Biospin Fungus Genomic DNA Extraction Kit-BSC14S1 (BioFlux ${ }^{\circledR}$, P.R. China); following the instructions of the manufacturer (Hangzhou, P.R. China). DNA sequence data was obtained from the internal transcribe spacer (ITS), large subunits of the nuclear ribosomal RNA genes (LSU). Primer sets used for these genes were as follows: ITS: ITS5/ITS4; LSU: LR0R/LR5 (Liu et al. 1999; Sung et al. 2007). The amplification was performed following the instructions, and were set up for initial denaturation of $5 \mathrm{~min}$ at $95^{\circ} \mathrm{C}$, followed by 35 cycles of $45 \mathrm{~s}$ at $94^{\circ} \mathrm{C}, 45 \mathrm{~s}$ at $52^{\circ} \mathrm{C}$ and $90 \mathrm{~s}$ at $72^{\circ} \mathrm{C}$, and a final extension period of $10 \mathrm{~min}$ at $72^{\circ} \mathrm{C}$. PCR-products were checked on $1 \%$ agarose electrophoresis gels stained with ethidium bromide. Purification and sequencing of PCR products were done by Majorbio Co., China. DNA sequence data were obtained from the large subunit rDNA (LSU) and internal transcribed spacers will amplify by primer pairs ITS5 and ITS4 (White et al. 1990). Primer sequences and database are available in GenBank. For Vagicola dactylidis single spore isolation was not successful. Therefore fungal DNA was isolated directly from the ascomata. 


\section{Phylogenetic analysis}

Sequences data were downloaded from GenBank to supplement the dataset (Table 1) (Phookamsak et al. 2014, Ariyawansa et al. 2015). The represented sequences including those newly obtained were aligned using with MAFFT v. 6.864b (http://mafft.cbrc.jp/alignment/server/index.html) and improved manually where necessary using Bioedit (Hall 1999). Didymella exigua was selected as outgroup taxon. The model of evolution was carried out using MrModeltest 2.2 (Nylander 2004). Maximum likelihood analysis was performed by using raxmlGUIv.0.9b2 (Silvestro and Michalak 2011). The search strategy was set to rapid bootstrapping and the analysis carried out using the GTRGAMMAI model of nucleotide substitution. The number of replicates was inferred using the stopping criterion (Pattengale et al. 2009). Maximum Likelihood bootstrap values equal or greater than $70 \%$ are given as the first set of numbers above the nodes (Fig. 1). PAUPv4.0b10 was used to conduct the parsimony analysis to obtain the phylogenetic trees. Trees were inferred using the heuristic search option with 1000 random sequence additions. Maxtrees were setup to 500 and branches of zero length were collapsed and all multiple parsimonious trees were saved. Descriptive tree statistics for parsimony (Tree Length [TL], Consistency Index [CI], Retention Index [RI], Relative Consistency Index [RC] and Homoplasy Index $[\mathrm{HI}]$ were calculated for trees generated under different optimality criteria. Kishino-Hasegawa tests (KHT) (Kishino and Hasegawa 1989) were performed in order to determine whether trees were significantly different. Maximum-parsimony bootstrap values equal or greater than $70 \%$ are given as the second set of numbers above the nodes (Fig. 1).

\section{Results and Discussion}

\section{Molecular phylogeny}

The combined LSU and ITS dataset comprising 57 strains of species of Phaeosphaeriaceae were used to determine the generic placement of our two strains as Vagicola dactylidis and $V$. chlamydospora. The phylogenetic trees obtained from Maximum Likelihood and Parsimony analysis yielded trees with similar overall topology at subclass and family relationships, in agreement with previous work based on Maximum Likelihood analysis (Zhang et al. 2012; Phookamsak et al. 2013, 2014, Ariyawansa et al. 2014a, b, c, 2015, Wijayawardene et al. 2013, Phukhamsakda et al. 2015). Individual LSU and ITS single gene trees were initially made and had a similar topology (data not shown). Therefore the genes were combined. The maximum parsimony dataset consists of 1360 characters with 987 characters as constant information, 113 characters as variable characters are parsimony-uninformative, and 260 characters were count as parsimonyinformative character. The most parsimonious tree showed $\mathrm{TL}=1873, \mathrm{CI}=0.343, \mathrm{RI}=0.613, \mathrm{RC}$ $=0.210, \mathrm{HI}=0.657$ values. The best scoring tree is presented in Figure 1. The strains of Vagicola dactylidis and V. chlamydospora clustered in the family Phaeosphaeriaceae. Vagicola dactylidis and V. chlamydospora formed a sister clade with V. vagans (CBS 604.86) with 52\% ML and 50\% MP support, but separate from other genera in the family. The new sequence data are deposited in GenBank (Table 1).

\section{Taxonomy}

Vagicola K.W.T. Chethana and K.D. Hyde, in Ariyawansa et al. Fungal Diversity (2015) = Phaeosphaeria subgen. Vagispora Shoemaker \& Babcock, Can. J. Bot. 67: 1500-1599 (1989)

Type species: Vagicola vagans (Niessl) O. Eriksson, Chethana \& K.D. Hyde, comb. nov. Basionym: Pleospora vagans Niessl, Verh. Naturf. Ver. Briinn 14: 174. 1876 $\equiv$ Phaeosphaeria vagans (Niessl) O.E. Erikss., Ark. Bot. 6: 430 (1967)

Vagicola chlamydospora Jayasiri, Camporesi \& K.D. Hyde, sp. nov. Fig. 2 Index Fungorum Number: IF551683

Facesoffungi Number: FoF 01323 


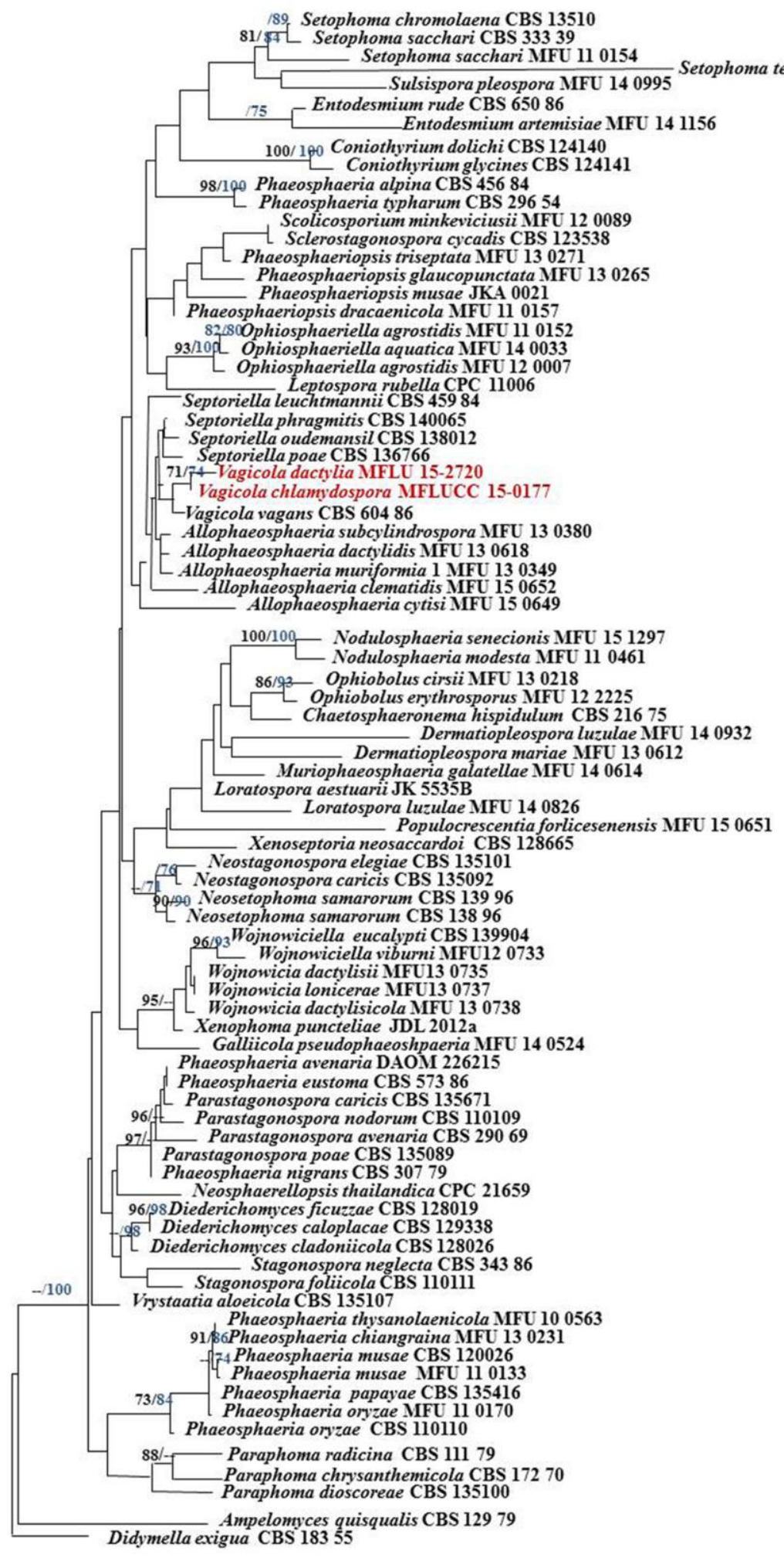

Ophiobolus

Fig. 1 - RAxML Maximum Likelihood phylogenetic tree based on a combined LSU and ITS sequence dataset. Bootstrap support values for Maximum Likelihood (ML) greater than $70 \%$ and Maximum-Parsimony bootstrap values above $70 \%$ are given above and below the nodes respectively. The tree is rooted to Didymella exigua (CBS 18355). 
Table 1 Taxa used in the phylogenetic analysis and GenBank accession numbers (LSU and ITS) and species. New sequences are in bold.

\begin{tabular}{|c|c|c|c|}
\hline \multirow[t]{2}{*}{ Taxon } & \multirow{2}{*}{$\begin{array}{c}\text { Voucher/culture } \\
\text { numbers }\end{array}$} & \multicolumn{2}{|c|}{ GenBank accession numbers } \\
\hline & & LSU & ITS \\
\hline Ampelomyces quisqualis & CBS 129.79 & EU754128 & HQ108038 \\
\hline Allophaeosphaeria cytisi & MFLUCC 15-0649 & KT306950 & KT306947 \\
\hline Allophaeosphaeria clematidis & MFLUCC 15-0652 & KT306953 & KT306949 \\
\hline Allophaeosphaeria dactylidis & MFLUCC 13-0618 & KP744473 & KP744432 \\
\hline Allophaeosphaeria subcylindrospora & MFLUCC 13-0380 & KT314183 & KT314184 \\
\hline Allophaeosphaeria muriformia & MFLUCC 13-0349 & KP765681 & KP765680 \\
\hline Chaetosphaeronema hispidulum & CBS 216.75 & KF251652 & KF251148 \\
\hline Coniothyrium dolichi & CBS 124140 & GQ387611 & JF740183 \\
\hline Coniothyrium glycines & CBS 124141 & KF251714 & KF251211 \\
\hline Dermatiopleospora mariae & MFLUCC 13-0612 & KJ749653 & KJ749654 \\
\hline Dermatiopleospora luzulae & MFLUCC 14-0932 & KT306951 & - \\
\hline Didymella exigua & CBS 183.55 & EU754155 & GU237794 \\
\hline Diederichomyces cladoniicola & CBS 128026 & JQ238628 & KP170642 \\
\hline Diederichomyces caloplacae & CBS 129338 & JQ238643 & KP170639 \\
\hline Diederichomyces ficuzzae & CBS 128019 & JQ238616 & KP170647 \\
\hline Entodesmium rude & CBS 650.86 & GU301812 & - \\
\hline Entodesmium artemisiae & MFLUCC 14-1156 & KT315509 & KT315508 \\
\hline Galliicola pseudophaeosphaeria & MFLUCC 14-0524 & KT326693 & KT326692 \\
\hline Leptospora rubella & CPC 11006 & DQ195792 & DQ195780 \\
\hline Loratospora aestuarii & JK 5535B & GU301838 & - \\
\hline Loratospora luzulae & MFLUCC 140826 & KT328495 & KT328497 \\
\hline Muriophaeosphaeria galatellae & MFLUCC 14-0614 & KT438329 & KT438333 \\
\hline Neosetophoma samarorum & CBS 139.96 & GQ387579 & KF251161 \\
\hline Neosetophoma samarorum & CBS 138.96 & KF251664 & KF251160 \\
\hline Neostagonospora caricis & CBS 135092 & KF251667 & KF251163 \\
\hline Neostagonospora elegiae & CBS 135101 & KF251668 & KF251164 \\
\hline Neosphaerellopsis thailandica & CPC 21659 & KP170721 & KP170652 \\
\hline Nodulosphaeria modesta & MFLUCC 11-0461 & KM434285 & KM434275 \\
\hline Nodulosphaeria senecionis & MFLUCC 15-1297 & KT290257 & KT290257 \\
\hline Ophiobolus cirsii & MFLUCC 13-0218 & KM014662 & KM014664 \\
\hline Ophiobolus erythrosporus & MFLUCC 12-2225 & KM014665 & KM491547 \\
\hline Ophiosphaerella aquatica & MFLUCC 14-0033 & & \\
\hline Ophiosphaerella agrostidis & MFLUCC 11-0152 & KM434281 & KM434271 \\
\hline Ophiosphaerella agrostidis & MFLUCC 12-0007 & KM434282 & KM434272 \\
\hline Paraphoma dioscoreae & CBS 135100 & KF251671 & KF251167 \\
\hline Paraphoma chrysanthemicola & CBS 172.70 & KF251669 & KF251165 \\
\hline Paraphoma radicina & CBS 111.79 & KF251676 & KF251172 \\
\hline Parastagonospora caricis & S6150/CBS135671 & KF251680 & KF251176 \\
\hline Parastagonospora nodorum & CBS 110109 & KF251681 & KF251177 \\
\hline Parastagonospora poae & CBS 135089 & KF251682 & KF251178 \\
\hline Phaeosphaeria papayae & CBS 135416 & KF251690 & KF251187 \\
\hline Phaeosphaeria alpina & CBS 456.84 & KF251684 & KF251181 \\
\hline Phaeosphaeria avenaria & DAOM 226215 & AY544684 & - \\
\hline
\end{tabular}




\begin{tabular}{|c|c|c|c|}
\hline \multirow[t]{2}{*}{ Taxon } & \multirow{2}{*}{$\begin{array}{c}\text { Voucher/culture } \\
\text { numbers }\end{array}$} & \multicolumn{2}{|c|}{ GenBank accession numbers } \\
\hline & & LSU & ITS \\
\hline Phaeosphaeria eustoma & CBS 573.86 & DQ678063 & - \\
\hline Phaeosphaeria chiangraina & MFLUCC 13-0231 & KM434280 & KM434270 \\
\hline Phaeosphaeria musae & MFLUCC 11-0133 & KM434277 & - \\
\hline Phaeosphaeria musae & CBS 120026 & GU301862 & DQ885894 \\
\hline Phaeosphaeria nigrans & CBS 307.79 & KF251687 & - \\
\hline Phaeosphaeria oryzae & CBS 110110 & KF251689 & KF251186 \\
\hline Phaeosphaeria oryzae & MFLUCC 11-0170 & KM434279 & KM434269 \\
\hline Phaeosphaeria thysanolaenicola & MFLUCC 10-0563 & KM434276 & KM434266 \\
\hline Phaeosphaeria typharum & CBS 296.54 & KF251695 & KF251192 \\
\hline Phaeosphaeria vagans & CBS 604.86 & KF251696 & KF251193 \\
\hline Phaeosphaeriopsis musae & CBS 120026 & GU301862 & DQ885894 \\
\hline Phaeosphaeriopsis dracaenicola & MFLUCC 11-0157 & KM434283 & KM434273 \\
\hline Phaeosphaeriopsis glaucopunctata & MFLUCC 13-0265 & KJ522477 & KJ522473 \\
\hline Phaeosphaeriopsis triseptata & MFLUCC 13-0271 & KJ522479 & KJ522475 \\
\hline Populocrescentia forlicesenensis & MFLUCC $15-0651$ & КT306952 & KT306948 \\
\hline Sclerostagonospora cycadis & CBS 123538 & FJ372410 & FJ372393 \\
\hline Scolicosporium minkeviciusii & MFLUCC 12-0089 & KF366382 & - \\
\hline Septoriella phragmitis & CBS 140065 & KR873279 & KR873251 \\
\hline Septoriella oudemansii & CBS 138012 & KJ869224 & KR873250 \\
\hline Septoriella poae & CBS 136766 & KJ869169 & KJ869111 \\
\hline Septoriella leuchtmannii & CBS 459.84 & KF251691 & KF251188 \\
\hline Setophoma achromolaena & $\begin{array}{c}\text { CBS } 135105 \mathrm{~T} / \mathrm{CPC} \\
18553\end{array}$ & KF251747 & KF251244 \\
\hline Setophoma sacchari & CBS 333.39 & KF251748 & KF251245 \\
\hline Setophoma sacchari & MFLUCC11-0154 & KJ476146 & KJ476144 \\
\hline Setophoma terrestris & CBS 335.29 & KF251749 & KF251246 \\
\hline Sulcispora pleospora & MFLUCC 14-0995 & & \\
\hline Stagonospora neglecta & CBS 343.86 & EU754218 & AJ496630 \\
\hline Stagonospora foliicola & CBS 110111 & KF251759 & KF251256 \\
\hline Vagicola chlamydospora & MFLUCC 15-0177 & KU163654 & KU163658 \\
\hline Vagicola dactylidis & MFLU 15-2720 & KU163656 & KU163657 \\
\hline Vagicola vagans & CBS 604.86 & KF251696 & KF251193 \\
\hline Vrystaatia aloeicola & CBS 135107 & KF251781 & KF251278 \\
\hline Wojnowiciella viburni & $\begin{array}{c}\text { MFLUCC 12-0733/ICMP } \\
19778\end{array}$ & KC594287 & KC594286 \\
\hline Wojnowicia dactylisicola & MFLUCC 13-0738 & KP684147 & KP744469 \\
\hline Wojnowicia dactylisii & MFLUCC 13-0735 & KP684149 & KP744470 \\
\hline Wojnowicia lonicerae & MFLUCC 13-0737 & KP684151 & KP744471 \\
\hline Wojnowiciella eucalypti & CBS 139904 & KR476774 & KR476741 \\
\hline Xenophoma puncteliae & JDL-2012a/CBS 128022 & JQ238619 & JQ238617 \\
\hline Xenoseptoria neosaccardoi & CBS 128665 & KF251784 & KF251281 \\
\hline
\end{tabular}

CBS Centraalbureau voor Schimmelcultures, Utrecht, The Netherlands; CPC Working collection of Pedro Crous housed at CBS; DAOM Plant Research Institute, Department of Agriculture (Mycology), Ottawa, Canada; ICMP International Collection of Microorganisms from Plants, New Zealand; JK: J. Kohlmeyer; MFLUCC Mae Fah Luang University Culture Collection, Chiang Rai, Thailand. 


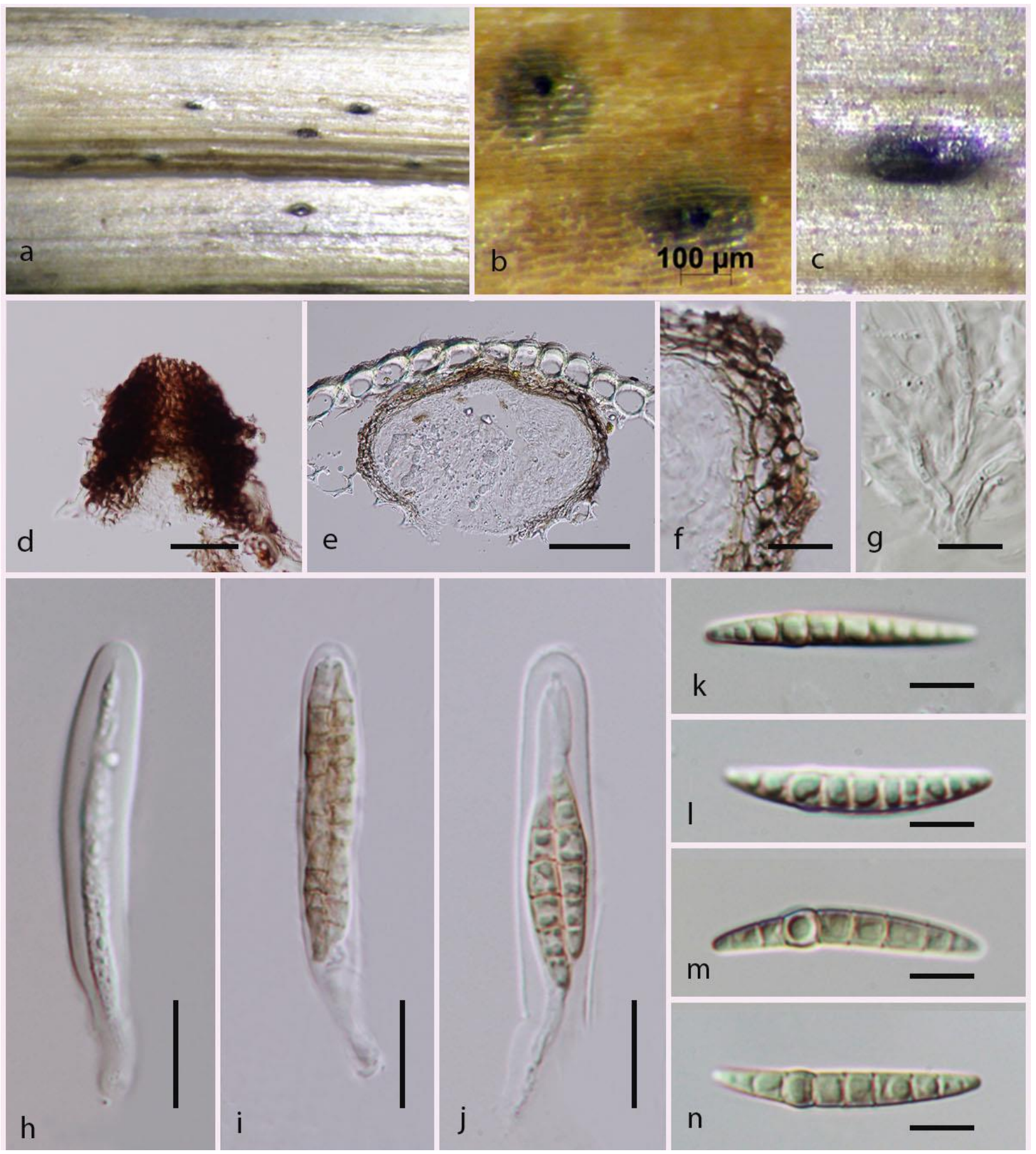

Fig. 2 - Vagicola chlamydospora (MFLU 15-1399, holotype) a-c Ascomata developing on surface of host. d Papilla. e Section through the ascoma. f Peridium. g Pseudoparaphyses. h-j Asci. k-n Ascospores. Scale bars: $d=30 \mu \mathrm{m}, \mathrm{e}=50 \mu \mathrm{m}, \mathrm{f}, \mathrm{h}-\mathrm{j}=10 \mu \mathrm{m}, \mathrm{g}, \mathrm{k}-\mathrm{n}=5 \mu \mathrm{m}$.

\section{Holotype - MFLU 15-1399}

Etymology - With reference to chlamydospores-like asexual morph formed in culture

Saprobic on Dactylis sp. Sexual morph: Ascomata 121-156 $\mu \mathrm{m}$ high, 177-208 $\mu \mathrm{m}$ diam., scattered or sometimes clustered, immersed, visible as raised, black dots on the host surface, uni- to bi-loculate, subglobose, brown to dark brown, ostiole central, with a minute papilla. Peridium 21$27 \mu \mathrm{m}$ wide, thin-walled, of equal thickness, composed of 2-5 layers of brown to dark brown, pseudoparenchymatous cells, arranged in textura angularis to inner layer composed of brown cells of textura prismatica. Hamathecium composed of numerous, 1.7-2.3 $\mu \mathrm{m}$ wide, filiform, broad, cellular pseudoparaphyses, with distinct septa, slightly constricted at the septa, embedded in a 

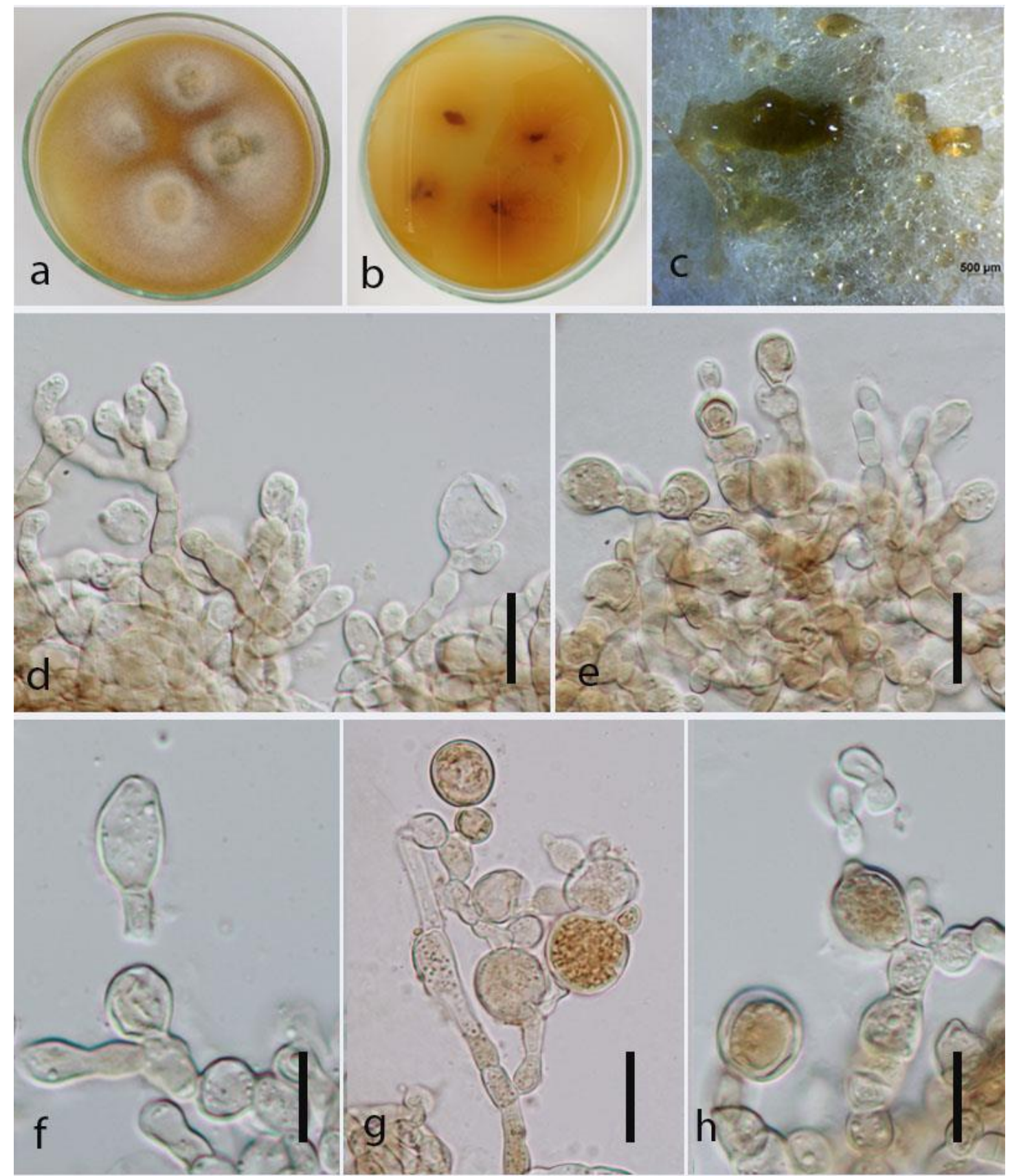

Fig. 3 - Vagicola chlamydospora asexual morph in culture (MFLUCC 15-0177, ex-type culture). a Fungal mycelium in MEA from above. b Fungal mycelium in MEA from below. c Close up view of fruiting body in culture. $d-h$ Asexual spores formation structures. Scale bars: $d-h=10 \mu \mathrm{m}$.

gelatinous matrix. Asci 54-57 ×9-10.6 $\mu \mathrm{m}(\mathrm{x}=57 \times 9.6 \mu \mathrm{m}, \mathrm{n}=20), 8$-spored, bitunicate, fissitunicate, broadly cylindrical or cylindric-clavate, sessile to subsessile, apically rounded with an ocular chamber. Ascospores $21-32 \times 3.7-5.7 \mu \mathrm{m}(\mathrm{x}=30 \times 4.6 \mu \mathrm{m}, \mathrm{n}=30)$, overlapping 1-3seriate, phragmosporous, narrowly fusiform, with obtuse ends, with guttules, hyaline to yellowishbrown, 9-septate, slightly curved, constricted at the septa, smooth-walled, lacking a sheath or appendages. Asexual morph: Colonies on MEA effuse, white to pale yellow. Mycelium 2-3 $\mu \mathrm{m}$ wide, prostrate, composed of septate, branched, smooth, hyaline, hyphae. Conidiophores micro- to macronematous, erect, flexuous, smooth, composed of beaded, variedly-sized cells. Conidiogenous cells 5-9 × 3-6 $\mu \mathrm{m}$ holoblastic, globose to subglobose, smooth, subhyaline. Conidia 9-12 × 10-13 $\mu \mathrm{m}(\mathrm{x}=10 \times 11 \mu \mathrm{m}, \mathrm{n}=20)$, globose, solitary, thick-walled, chlamydospore-like, with dense cytoplasm (Fig 3). 

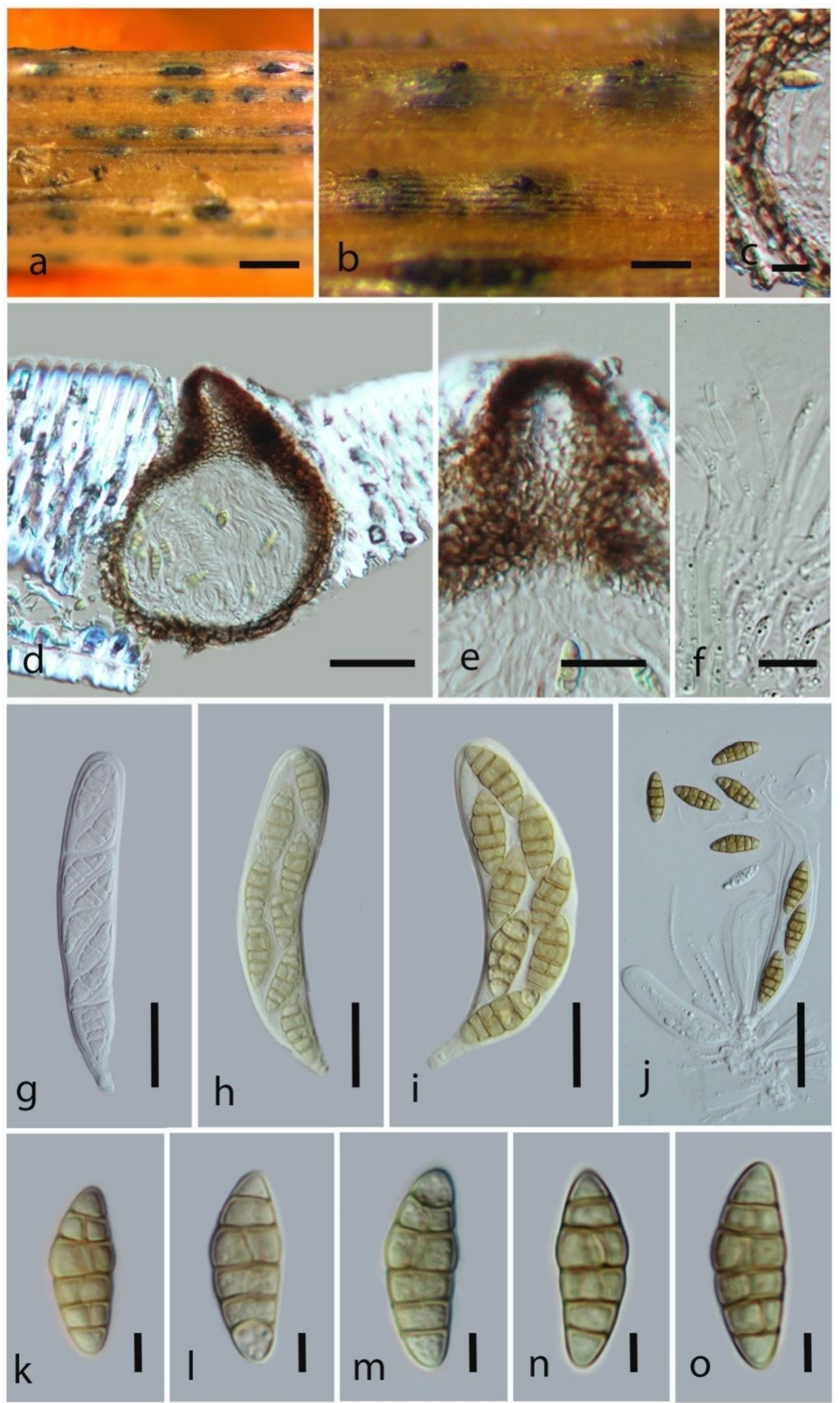

Fig. 4 - Vagicola dactylidis (holotype). a, b Ascomata developing on surface of host. c Peridium. d Section through ascoma e Papilla. f Pseudoparaphyses. g-j Asci. k-o Ascospores. Scale bars: $\mathrm{a}=$ $500 \mu \mathrm{m}, \mathrm{b}=200 \mu \mathrm{m}, \mathrm{c}=10 \mu \mathrm{m}, \mathrm{d}=50 \mu \mathrm{m}, \mathrm{e}=20 \mu \mathrm{m}, \mathrm{g}-\mathrm{j}=20 \mu \mathrm{m}, \mathrm{g}, \mathrm{f}, \mathrm{k}-\mathrm{o}=5 \mu \mathrm{m}$. 
Material examined - ITALY, Province of Forlì-Cesena, near Poderone - Corniolo, dead stem of Dactylis sp. (Poaceae), 21 October 2014, Erio Camporesi IT 2188 (MFLU 15-1399, holotype), (isotype in KUN), ex-type culture, MFLUCC 15-0177, Genbank accession numbers: LSU- KU163654, ITS-KU163658, SSU-KU163655.

Culture characters - Ascospores germinating on MEA within $36 \mathrm{~h}$. Colonies growing on MEA, reaching $2 \mathrm{~cm}$ diam. in 1 week at $16^{\circ} \mathrm{C}$. Mycelium superficial, felty, gummy, edge undulate, from above white, reverse yellow colour.

Notes - Vagicola chlamydospora resembles to Vagicola vagans in having similar ascomata, as black coloured dots of ostiole visible with immersed ascomata when viewed on the host surface, textura angularis to textura prismatica brown colour cells peridium. and broad cellular pseudoparaphyses but differs in processing longitudinal septa and narrow ascospores. The spores in Vagicola chlamydospora are similar to Nodulosphaeria, but differ in the morphology of ascomata and phylogenetic analyses. In this study we observed chlamydospore-like asexual morph in culture (Fig. 3).

Vagicola dactylidis Wanasinghe, Jayasiri, Camporesi \& K.D. Hyde, sp. nov.

Fig. 4 Index Fungorum Number: IF551684

Facesoffungi Number: FoF 01324

Holotype - MFLU 15-2720

Etymology - With reference to the host occurrence

Saprobic on dead stem of Dactylis sp. Sexual morph: Ascomata 120-180 $\mu \mathrm{m}$ high, 110-160 $\mu \mathrm{m}$ diam. $(\overline{\mathrm{x}}=153.9 \times 141.3 \mu \mathrm{m}, \mathrm{n}=10)$, solitary, scattered, superficial, globose to subglobose, dark brown to black, coriaceous, ostiolate. Ostiole 50-60 $\mu \mathrm{m}$ high, $20-30 \mu \mathrm{m}$ diam. $(\overline{\mathrm{x}}=55.5 \times 27.5$ $\mu \mathrm{m}, \mathrm{n}=5$ ), papillate, black, smooth, filled with dark brown cells. Peridium 9-12 $\mu \mathrm{m}$ wide at the base, 12-16 $\mu \mathrm{m}$ wide in sides, comprising 3-4 layers, comprising blackish to dark brown, thickwalled cells of textura angularis. Hamathecium comprising numerous, $2-2.5 \mu \mathrm{m}(\mathrm{n}=30)$ wide, filamentous, branched, septate, pseudoparaphyses. Asci 70-120 $\times 15-21 \mu \mathrm{m}(\overline{\mathrm{x}}=84.4 \times 18.7 \mu \mathrm{m}, \mathrm{n}$ $=40$ ), 8 -spored, bitunicate, fissitunicate, broadly-clavate, with a short, orbicular pedicel, rounded at apex, with minute ocular chamber. Ascospores 19-23 $\times 6-9 \mu \mathrm{m}(\overline{\mathrm{x}}=20.7 \times 7.4 \mu \mathrm{m}, \mathrm{n}=50)$, obliquely bi-seriate, initially hyaline, becoming yellowish brown at maturity, broadly fusiform, 5trans-septate, with a longitudinal septa in between second and third trans-septate, constricted at the central septa, weakly constricted at the other septa, with conical and narrowly rounded ends, lacking a mucilaginous sheath. Asexual morph: Undetermined.

Material examined - ITALY, Province of Arezzo, Bagno di Cetica, dead stem of Dactylis sp. (Poaceae), 8 October 2012, Erio Camporesi. IT 799 (MFLU 15-2720, holotype), (isotype in KUN), GenBank accession numbers: LSU - KU163656, ITS - KU163657. Notes - Vagicola dactylidis is more similar to the type species, V. vagans, than $V$. chlamydospora, in having ascospores with vertical septa. Dactylis is the host plant of both Vagicola chlamydospora and dactylidis. Two other species associated with Dactylis in Phaeosphaeriaceae are Phaeosphaeria huronensis (Shoemaker \& Babcock 1989) and Ophiosphaerella herpotricha (Phookamsak et al. 2014), however, they share no morphological characters.

\section{Acknowledgements}

This work was supported by the International Research Group Program (IRG-14-27), Deanship of Scientific Research, King Saud University, Saudi Arabia. K.D. Hyde thanks the Chinese Academy of Sciences, [project number 2013T2S003], for the award of Visiting Professorship for Senior International Scientists at Kunming Institute of Botany. MFLU [grant number 56101020032] is thanked for supporting studies on Dothideomycetes. We are grateful to the Mushroom Research Foundation, Chiang Rai, Thailand. Subashini C. Jayasiri is grateful to Mr. and (Late) Mrs. Jayasiri and S.P.R.D. Lasantha for their valuable suggestions. 


\section{References}

Ariyawansa HA, Hyde KD, Jayasiri SC, Buyck B, Chethana KWT, Dai DQ, Dai YC, Daranagama DA, Jayawardena RS, Lücking R, Ghobad-Nejhad M, Niskanen T, Thambugala KM, Voigt K, Zhao RL, Li GJ, Doilom M, Boonmee S, Yang ZL, Cai Q, Cui YY, Bahkali AH, Chen J, Cui BK, Chen JJ, Dayarathne MC, Dissanayake AJ, Ekanayaka AH, Hashimoto A, Hongsanan S, Jones EBG, Larsson E, Li WJ, Li QR, Liu JK, Luo ZL, Maharachchikumbura SSN, Mapook A, McKenzie EHC, Norphanphoun C, Konta S, Pang KL, Perera RH, Phookamsak R, Phukhamsakda C, Pinruan U, Randrianjohany E, Singtripop C, Tanaka K, Tian CM, Tibpromma S, Abdel-Wahab MA, Wanasinghe DN, Wijayawardene NN, Zhang JF, Zhang H, Abdel-Aziz FA, Wedin M, Westberg M, Ammirati JF, Bulgakov TS, Lima DX, Callaghan TM, Callac P, Chang CH, Coca LF, Dal-Forno M, Dollhofer V, Fliegerová K, Greiner K, Griffith GW, Ho HM, Hofstetter V, Jeewon R, Kang JC, Wen TC, Kirk PM, Kytövuori I, Lawrey JD, Xing J, Li H, Liu ZY, Liu XZ, Liimatainen K, Lumbsch HT, Matsumura M, Moncada B, Nuankaew S, Parnmen S, de Azevedo Santiago ALCM, Sommai S, Song Y, de Souza CAF, de Souza-Motta CM, Su HY, Suetrong S, Wang Y, Wei SF, Wen TC, Yuan HS, Zhou LW, Réblová M, Fournier J, Camporesi E, Luangsa-ard JJ, Tasanathai K, Khonsanit A, Thanakitpipattana D, Somrithipol S, Diederich P, Millanes AM, Common RS, Stadler M, Yan JY, Li XH, Lee HW, Nguyen TTT, Lee HB, Battistin E, Marsico O, Vizzini A, Vila, J, Ercole E, Eberhardt U, Simonini G, Wen HA, Chen XH. 2015 - Fungal Diversity Notes 111-252-taxonomic and phylogenetic contributions to fungal taxa. Fungal Diversity 75(1) (DOI 10.1007/s13225-015-0346-5)

Ariyawansa H, Phookamsak R, Tibpromma S, Kang JC, Hyde KD. 2014a - A molecular and morphological reassessment of Diademaceae. The Scientific World Journal 2014 1-11 (DOI 10.1155/2014/675348)

Ariyawansa HA, Camporesi E, Thambugala KM, Mapook A, Kang JC, Alias SA, Chukeatirote E, Thines M, McKenzie EHC, Hyde KD 2014b - Confusion surrounding Didymosphaeria phylogenetic and morphological evidence suggest Didymosphaeriaceae is not a distinct family. Phytotaxa 176, 102-119 (DOI 10.11646/phytotaxa.181.1.1)

Ariyawansa HA, Kang JC, Alias SA, Chukeatirote E, Hyde KD 2014c - Pyrenophora. Mycosphere 5, 351-362 (DOI 10.5943/mycosphere/5/2/9)

Barr ME. 1979 - A classification of Loculoascomycetes. Mycologia 71，935-957 (DOI $10.2307 / 3759283)$

Cannon PF, Kirk PM. 2007 - Fungal Families of the World. CAB. International, Wallingford, Oxfordshire, U.K.

Carson ML. 2005 - Yield loss potential of Phaeosphaeria leaf spot of maize caused by Phaeosphaeria maydis in the United States. Plant Disease 89, 986-988 (DOI 10.1094/PD-89-0986)

Chomnunti P, Hongsanan S, Hudson BA, Tian Q, Persoh D, Dhami MK, Alias AS, Xu J, Liu X, Stadler M, Hyde, KD. 2014 - The Sooty Moulds. Fungal Diversity 66, 1-36 (DOI 10.1007/s13225-014-0278-5)

De Gruyter J, Woudenberg JH, Aveskamp MM, Verkley GJ, Groenewald JZ, Crous PW. 2010 Systematic reappraisal of species in Phoma section Paraphoma, Pyrenochaeta and Pleurophoma. Mycologia 102, 1066-1081 (DOI 10.3852/09-240)

Eriksson O. 1967 - On graminicolous Pyrenomycetes from Fennoscandia 2. Phragmosporous and scolecosporous species. Arkiv för Botanik, serie 2, 6, 381-440.

Hyde KD, Jones EBG, Liu JK, Ariyawansa H, Boehm E, Boonmee S, Braun U, Chomnunti P, Crous PW, Dai DQ, Diederich P, Dissanayake A, Doilom M, Doveri F, Hongsanan S, Jayawardena R, Lawrey JD, Li YM, Liu YX, Lücking R, Monkai J, Muggia L, Nelsen MP, Pang KL, Phookamsak R, Senanayake I, Shearer CA, Suetrong S, Tanaka K, Thambugala KM, Wijayawardene NN, Wikee S, Wu HX, Zhang, Y, Aguirre-Hudson B, Alias SA, Aptroot A, Bahkali AH, Bezerra JL, Bhat DJ, Camporesi E, Chukeatirote E, Gueidan C, 
Hawksworth DL, Hirayama K, Hoog SD, Kang JC, Knudsen K, Li WJ, Li XH, Liu ZY, Mapook A, McKenzie EHC, Miller AN, Mortimer PE, Phillips AJL, Raja HA, Scheuer C, Schumm F, Taylor JE, Tian Q, Tibpromma S, Wanasinghe DN, Wang Y, Xu JC, Yan JY, Yacharoen S, Zhang M. 2013 - Families of Dothideomycetes. Fungal Diversity 63, 1-313 (DOI 10.1007/s13225-013-0263-4)

Index Fungorum (2015) http://www.indexfungorum.org/Names/Names. asp. Accessed on November 2015

Jayasiri SC, Hyde KD, Ariyawansa HA, Bhat J, Buyck B, Cai L, Dai YC, Abd-Elsalam KA, Ertz D, Hidayat I, Jeewon R, Jones EBG, Bahkali AH, Karunarathna SC, Liu JK, Luangsa-ard JJ, Lumbsch HT, Maharachchikumbura SSN, McKenzie EHC, Moncalvo, JM, GhobadNejhad M, Nilsson H, Pang KA, Pereira OL, Phillips AJL, Raspé O, Rollins AW, Romero AI, Etayo J, Selçuk F, Stephenson SL, Suetrong S, Taylor JE, Tsui CKM, Vizzini A, Abdel-Wahab MA, Wen TC, Boonmee S, Dai DQ, Daranagama DA, Dissanayake AJ, Ekanayaka AH, Fryar SC, Hongsanan S, Jayawardena RS, Li WJ, Perera RH, Phookamsak R, de Silva NI, Thambugala KM, Tian Q, Wijayawardene NN, Zhao RL, Zhao Q, Kang JC, Promputtha I. 2015 - The Faces of Fungi database: fungal names linked with morphology, phylogeny and human impacts. Fungal Diversity 74 (DOI 10.1007/s13225015-0351-8)

Kishino H, Hasegawa M. 1989 - Evaluation of the maximum likelihood estimate of the evolutionary tree topologies from DNA - sequence data, and the branching order in Hominoidea. Journal of Molecular Evolution 29, 170-179 (DOI 10.1007/BF02100115)

Hall TA. 1999 - BioEdit: a user-friendly biological sequence alignment editor and analysis program for Windows 95/98/NT. Nucleic Acids Symposium Series 41, 95-98.

Liu YJ, Whelen S, Hall BD. 1999 - Phylogenetic relationships among ascomycetes: evidence from an RNA polymerse II subunit. Molecular Biology and Evolution 16, 1799-1808.

Miyake I. 1909 - Studies on the parasitic fungi of rice in Japan. Botanical Magazine Tokyo. 23: 85-97.

Nylander JAA. 2004 - MrModeltest 2.0. Program distributed by the author. Evolutionary Biology Centre, Uppsala University

Pattengale ND, Alipour M, Bininda-Emonds ORP, Moret BME, Stamatakis A. 2009 - How many bootstrap replicates are necessary?. LNCS 5541, 184-200

Phookamsak R, Liu JK, Chukeatirote E, McKenzie EHC, Hyde KD. 2013 - Phylogeny and morphology of Leptosphaerulina saccharicola sp. nov. and Pleosphaerulina oryzae and relationships with Pithomyces. Cryptogamie Mycologie 34, 303-319 (10.7872/crym.v34.iss4.2013.303)

Phookamsak R, Liu JK, McKenzie EHC, Manamgoda DS, Ariyawansa HA, Thambugala, KM, Dai DQ, Camporesi E, Chukeatirote E, Wijayawardene NN, Bahkali AH, Mortimer PE, Xu JC, Hyde KD 2014 - Revision of Phaeosphaeriaceae. Fungal Diversity 68, 159-238 (DOI 10.1007/s13225-014-0308-3)

Phookamsak R, Manamgoda DS, Li WJ, Dai DQ, Singtripop C, Hyde KD 2015 - Poaceascoma helicoides gen et sp. nov., a new genus with scolecospores in Lentitheciaceae. Cryptogamie Mycologie 36(2), 1-12 (DOI 10.7872/crym/v36.iss2.2015.225)

Phukhamsakda C, Ariyawansa HA, Phookamsak R, Chomnunti P, Bulgakov TS, Yang JB, Bhat DJ, Bahkali AH, Hyde KD. 2015 - Muriphaeosphaeria galatellae gen. et sp. nov. in Phaeosphaeriaceae (Pleosporales). Phytotaxa 227, 55-65 (DOI 10.11646/phytotaxa.227.1.6)

Schoch CL, Shoemaker RA, Seifert KA, Hambleton S, Spatafora JW, Crous PW. 2006 - A multigene phylogeny of the Dothideomycetes using four nuclear loci. Mycologia 98, 10411052

Shoemaker RA, Babcock CE. 1989 - Phaeosphaeria. Canadian Journal of Botany 67, 1500-1599 (DOI 10.1139/b89-199) 
Silvestro D, Michalak I. 2011 - raxmlGUI: a graphical front-end for RAxML. Organisms Diversity \& Evolution 12 (4), 335-337 (DOI 10.1007/s13127-011-0056-0)

Stukenbrock EH, Banke S, McDonald BA. 2006 - Global migration patterns in the fungal wheat pathogen Phaeosphaeria nodorum. Molecular Ecology 15, 2895-2904 (DOI 10.1111/j.1365-294X.2006.02986.x)

Sung GH, Sung JM, Hywel Jones NL, Spatafora JW. 2007 - A multigene phylogeny of Clavicipitaceae (Ascomycota, fungi): identification of localized incongruence using a combinational bootstrap approach. Molecular Phylogenetics \& Evolution 44, 1204-1223.

White TJ, Bruns TD, Lee S, Taylor J. 1990 - Amplification and direct sequencing of fungal ribosomal RNA genes for phylogenetics. PCR Protocols: Guide Methods Application 18, 315-322 (10.1016/B978-0-12-372180-8.50042-1)

Wijayawardene DNN, Camporesi E, Song Y, Dai DQ, Bhat DJ, McKenzie EHC, Chukeatirote E, Mel'nik VA, Wang Y, Hyde KD. 2013 - Multi-gene analyses reveal taxonomic placement of Scolicosporium minkeviciusii in Phaeosphaeriaceae (Pleosporales). Cryptogamie Mycologie 34, 357-366 (DOI 10.7872/crym.v35.iss2.2014.105)

Wijayawardene NN, Crous PW, Kirk PM, Hawksworth DL, Boonmee S, Braun U, Dai DQ D'souza MJ, Diederich P, Dissanayake A, Doilom M, Hongsanan S, Jones EBG, Groenewald JZ, Jayawardena R, Lawrey JD, Liu JK, Lücking R, Madrid H, Manamgoda DS, Muggia L, Nelsen MP, Phookamsak R, Suetrong S, Tanaka K, Thambugala KM, Wanasinghe DN, Wikee S, Zhang Y (24), Aptroot A, Ariyawansa HA, Bahkali AH, Bhat DJ, Gueidan C, Chomnunti P, De Hoog GS, Knudsen K, Li WJ, McKenzie EHC, Miller AN, Phillips AJL, Piątek M, Raja HA, Shivas RS, Slippers B, Taylor JE, Tian Q, Wang Y, Woudenberg JHC, Cai L, Jaklitsch WM, Hyde KD. 2014 - Naming and outline of Dothideomycetes 2014 - Fungal Diversity 69(1), 1-55 (DOI 10.1007/s13225-014-0309-2)

Zhang Y, Schoch CL, Fournier J, Crous PW, de Gruyter J, Woudenberg JHC, Hirayama K, Tanaka K, Pointing SB, Spatafora JW, Hyde KD. 2009 - Multi-locus phylogeny of Pleosporales: a taxonomic, ecological and evolutionary re-evaluation. Studies in Mycology 64, 85-102 (DOI 10.3114/sim.2009.64.04)

Zhang Y, Crous PW, Schoch CL, Hyde KD. 2012 - Pleosporales. Fungal Diversity 53, 1-221 (DOI 10.1007/s13225-011-0117-x) 